\title{
Realization of Maneuver trajectory in Computer wargaming system based on an Improved $A^{*}$ Algorithm
}

\author{
Rong Yang ${ }^{1,}$, ChangJun $\mathrm{Li}^{1, \text { a }}$, Zhangzhi Tao ${ }^{1, \text { a }}$ \\ ${ }^{1}$ The 28th Institute of CETC, Nanjing, China \\ arongyang@mail.ustc.edu.cn
}

Keywords: Maneuver trajectory, Computer wargaming system, Trajectory planning, Improved $A^{*}$ Algorithm.

\begin{abstract}
The traditional Computer wargaming system just considers a few types of simple landform, and maneuverable rules are also simple. Based on that, the maneuver trajectory planning results can be usable for games. But the results may be quite different from real combat, which causes simulation results be distrusted. To solve the above problems, this paper does trajectory planning based on real landform and real maneuverable rules. The traditional trajectory planning usually takes Euclid distance or coordinate difference as heuristic function which cannot satisfy the requirement of hexagon map. To use the characteristics of hexagonal grids, this paper takes a new heuristic function. The new method improves the efficiency and gets the best results. At last, this paper gives some simulation for different cases. And the simulation results show that the new algorithm is effective and reasonable.
\end{abstract}

\section{Introduction}

Wargame was invented by Von les Horowitz at 1811, who was consultant for Prussia. Wargame are composed of chessboard, chessman and rules [1]. Wargaming is application of wargame. Based on martial rules and probability theory, wargaming simulates battle process and investigates the impact of uncertainty and chanciness to battle results. So wargaming is the best tools to explore battle process [2, 3]. Computer wargaming system is one type of special war simulation system. The system summarizes human's knowledge and experience about battle disciplinarian as wargame rules, and does computer simulation by suing system emulator The Commanders' wisdom and experience will be fully utilized by using the computer wargaming system [4].

In traditional computer wargaming system and manual wargame, they just consider a few types of simple landform, which are sea, plain, highland, forest, rives and road [5], and the maneuverable rules are also simple. Based on that, the maneuver trajectory planning results are also relative simple. The real landform, physiognomy and maneuver rules are more complicated. For example, sea can be divided into blue water, shallow sea and bank. For real battle, their impacts on trajectory planning are quite different, because marine can sail at blue water and partial shallow sea while they cannot sail at bank. To make the computer wargaming system results useful and referenced for real battle, this paper considers many different real landforms, and does trajectory planning according to real maneuver rules. The simulation results can be used for reference to investigate battle.

\section{Wargaming Models}

Chessboard. Wargame chessboard is called wargame map denoting different landforms. Each chessboard grid has its landform and geographical information which affects battle operation, such as road, bridge, gulch, cliff, port and so on. When denoting real or suppositional map, hexagon grids or square grids can be used. Since hexagon grids have better consistency than square grids with the same sampling density [6], Hexagon grids are the most popular and taken in this paper. To differentiate 
landform affect wargaming differently, this paper divided landform into blue water, shallow sea, bank, plain, hill, lake, highland, desert, forest, bramble and city, which are denoted by different color and figures in Fig. 1.

Besides the impact of landform, river, road, railway, bridge, gulch, cliff also affect the results of wargaming. For example, infantry cannot cross river directly while they can march quickly on road.

To differentiate the impact of different factors, geography information about road and bridge should be denoted on wargame map. After denoting wargame map by hexagon grids, chessman is located at the center of one hexagon grid, and chessman's real position is denoted by the position of the hexagon grid. To denote the impact of river, road, railway, bridge, gulch and cliff to chessman, it is necessary to correct the position of the real geography information on wargame map according to the real terrain information. For example, river, bridge, gulch, cliff and border are described along the edge of hexagon grids, this is convenient for deciding if the army crosses river, bridge, gulch, cliff or border when they enter one grid from the other grid. Railway and road are described along centers of hexagon grids since chessman is located at the center of hexagon grids. Army marches by road when chessman moves between two road hexagon grids as in Fig. 2.

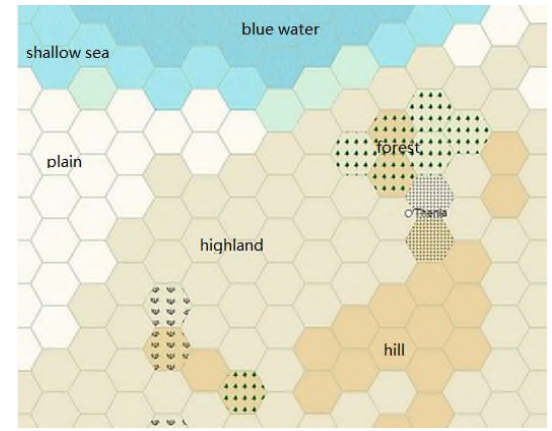

Fig. 1 Landform map.

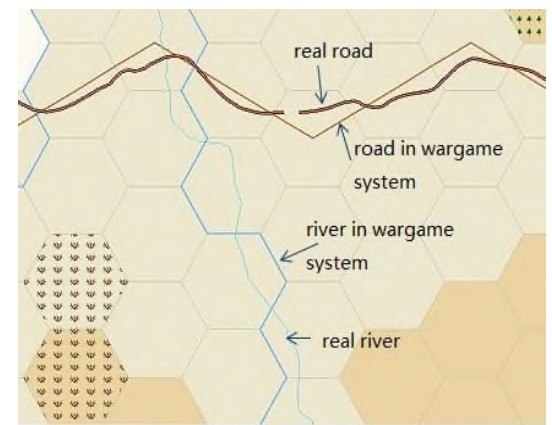

Fig. 2 Road and river map.

Chessman. Chessman of wargame denotes battle unit (army or weapon platform). The color of chessman denotes side of army (red side or blue side). There are main capability parameters on chessman, which depend on weapon performance, training level of army and battle experiences. A chessman can denote one brigade, one fight unit, one battalion or one plane, which depends on real application. The information including attack capability, recovery capability, maneuver capability, army type, equipment, battle code, designation, target type, weapon level and so on is described on chessman as in Fig. 3.

Wargame Rules. Wargame rules are the basic rules of wargaming. Wargame rules are from summarization of history battle and dry running data. They reflect military idea, battle theory, command trick and weapon using [7]. Wargame rules usually include distribution rules, marching rules, order rules and judging rules. Distribution rules decide how to distribute chessman on chessboard. 
Marching rules explain how different chessman move including maneuver, engagement, logistics and support. Judging rules calculate and judge the results of battle.

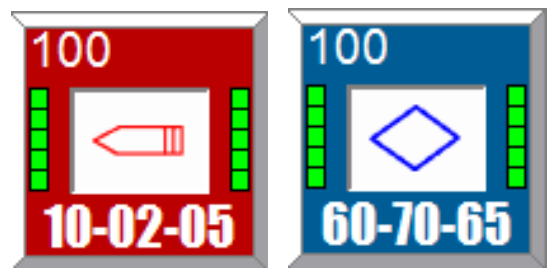

Fig. 3 Chessman units.

\section{Chessman Maneuver and Algorithm}

Maneuver is one of the most three important factors of battle. By maneuvering, battle unit can transmit force and weapons [8]. Maneuver is basis of battle and throughout the whole battle process. The purpose of battle operation is hold space, and maneuver is the basic and final method to hold space [9]. Maneuver unit is battalion or company battle unit, and there are four types of maneuver which are by foot, by car, by tank and by lift.

Maneuver Speed. Maneuver speed of battle unit changes with different landform, and is also impacted by maneuver way, weather, tire level, fire and so on [10]. This paper corrects maneuver speed to denote the impact of the above factors as in Table 1 .

When battle unit maneuvers, its speed decreases because of bad weather. This paper corrects maneuver speed according to weather as follows: maneuver speed decreases by $60 \%$ when visibility is less than 100 meters; maneuver speed decreases by $40 \%$ when visibility is less than 200 meters. Maneuver speed by foot decreases by $30 \%$ and maneuver speed by car or by tank decreases by $40 \%$ for median rain; maneuver speed by foot decreases by $45 \%$ and maneuver speed by car or by tank decreases by $50 \%$ for downfall; maneuver speed by foot decreases by $65 \%$ and maneuver speed by car or by tank decreases by $60 \%$ for rainstorm. Maneuver speed by foot decreases by $35 \%$ and maneuver speed by car or by tank decreases by $20 \%$ when it is blustery and direction of wind is contrary to maneuver direction. Tire level of unit affects speed of maneuver by foot, and don't affect speed of maneuver by car or by tank. This paper corrects maneuver speed according to tire level as follows: maneuver speed don't change of tire level 1 and level 2; maneuver speed decreases by $25 \%$ for tire level 3, maneuver speed decreases by $40 \%$ for tire level 4 . This paper corrects maneuver speed according to fire as follows: maneuver speed by foot decreases $40 \%$ and maneuver speed by car or tank decreases $20 \%$ during maneuver when fired by air force or by artillery from the other side.

Table 1. Maneuver speed correction for landform.

\begin{tabular}{|c|c|c|c|}
\hline \multirow{2}{*}{ Landform } & \multicolumn{3}{|c|}{ Correct coefficient } \\
\cline { 2 - 4 } & $\boldsymbol{B y}$ foot & By cay & By tank \\
\hline Plain & - & - & - \\
\hline Desert & $20 \%$ & $25 \%$ & $10 \%$ \\
\hline Highland & - & - & - \\
\hline Hill & $/$ & $/$ & $/$ \\
\hline Forest & $/$ & $/$ & $/$ \\
\hline Road & - & - & - \\
\hline Bridge & - & - & - \\
\hline River & $20 \%$ & $15 \%$ & $10 \%$ \\
\hline Cliff & $50 \%$ & $/$ & $/$ \\
\hline Gulch & $10 \%$ & $/$ & $20 \%$ \\
\hline
\end{tabular}


Maneuver Trajectory Planning. Trajectory planning is quite important in computer wargaming system. It is basic capability of all movable units. Trajectory planning is a challenge mission because of complexity of landform, tremendous number of hexagon grids, maneuver rules' complexity, realtime requirement and constriction of compute source. This paper doesn't consider the case of maneuver of chessman when doing trajectory planning. When more than one unit moves together, the speed of the whole units is calculated as the slowest unit.

Heuristic searching method is usually taken when doing trajectory planning. And A* algorithm is the most taken and the most advanced method in trajectory planning [11]. It is one dynamic heuristic searching method. And the algorithm finds the best trajectory by approaching destination using evaluate function as constraint condition. The most important thing using $\mathrm{A}^{*}$ algorithm is to find appropriate evaluate function and to expand grids.

Heuristic function (distance between any two hexagon grid (x1, y1) and (x2, y2)) affects efficiency and results of trajectory planning directly. And it will be better when taking more approximate heuristic function. At present, Euclid distance $\left(\mathrm{h}=\operatorname{sqrt}\left((\mathrm{x} 1-\mathrm{x} 2)^{\wedge} 2+(\mathrm{y} 1-\mathrm{y} 2)^{\wedge} 2\right)\right.$ or coordinate difference $(\mathrm{h}=\mathrm{x} 1-$ $\mathrm{x} 2+\mathrm{y} 1-\mathrm{y} 2)$ are usually taken as the heuristic function. But the above heuristic function cannot satisfy the requirement of hexagon map in computer wargaming system. Therefore, according to characteristic of hexagon grid, this paper gives the following heuristic functions:

(1) $\mathrm{x} 1$ and $\mathrm{x} 2$ are both odd or even

(2) $x 1$ is odd, $x 2$ is even, and $y 1>=y 2$

$$
\mathrm{h}=\max \{|\mathrm{x} 1-\mathrm{x} 2|,|\mathrm{y} 1-\mathrm{y} 2|+|\mathrm{x} 1-\mathrm{x} 2| / 2\}
$$

(3) $\mathrm{x} 1$ is odd, $\mathrm{x} 2$ is even, and $\mathrm{y} 1<\mathrm{y} 2$

$$
\mathrm{h}=\max \{|\mathrm{x} 1-\mathrm{x} 2|,|\mathrm{y} 1-\mathrm{y} 2|+(|\mathrm{x} 1-\mathrm{x} 2|+1) / 2\}
$$

(4) $x 1$ is even, $x 2$ is odd, and $y 1>=y 2$

$$
\mathrm{h}=\max \{|\mathrm{x} 1-\mathrm{x} 2|,|\mathrm{y} 1-\mathrm{y} 2|+(|\mathrm{x} 1-\mathrm{x} 2|-1) / 2\}
$$

(5) $\mathrm{x} 1$ is even, $\mathrm{x} 2$ is odd, and $\mathrm{y} 1<\mathrm{y} 2$

$$
\mathrm{h}=\max \{|\mathrm{x} 1-\mathrm{x} 2|,|\mathrm{y} 1-\mathrm{y} 2|+(|\mathrm{x} 1-\mathrm{x} 2|-1) / 2\}
$$

$$
\mathrm{h}=\max \{|\mathrm{x} 1-\mathrm{x} 2|,|\mathrm{y} 1-\mathrm{y} 2|+(|\mathrm{x} 1-\mathrm{x} 2|+1) / 2\}
$$

When expanding grids, it is necessary to judge if one chessman can move from the present grid to its adjacent grid. That means it is necessary to judge the attribute of maneuver chessman, if there is chessman on its adjacent grid and the chessman's attribute, if the adjacent grid is one forbidden grid, if the adjacent grid is obstacle and so on.

When a chessman enter its adjacent grid, the attribute of chessman should satisfy the following rules: one chessman cannot enter its adjacent grid if there is enemy chessman on the grid; one chessman can enter its adjacent grid if there is friendly chessman on the grid and less than three chessmen are on the grid. Forbidden grid is area which cannot pass through, such as enemy mines found by reconnaissance. Rules about landform are as follows: one chessman can pass through desert, settlement place, plain, highland, and its speed should be corrected according to different landform. Rules about grid edges are as follows: if there is gulch, cliff or river, one chessman can pass through the edge only when there is bridge on the edge.

This paper did trajectory planning according to the above heuristic function and rules when expanding grids.

\section{Simulation}

Operating process of trajectory planning is as follows: choose one chessman, click button Mov, and choose one destination grid. Then computer wargaming system will do trajectory planning and results will be shown using continuous arrows as in Fig. 4 and Fig. 5. Fig. 4 shows effectiveness of trajectory planning because the chessman arrives at destination grid by the only road in hills. Fig. 5 shows rationality of trajectory planning because the chessman cannot pass through river when there is no road 
or bridge, and arrives at destination choosing fast plain instead of slow road on hills. When there is no trajectory to destination, system will give hint as in Fig. 6.

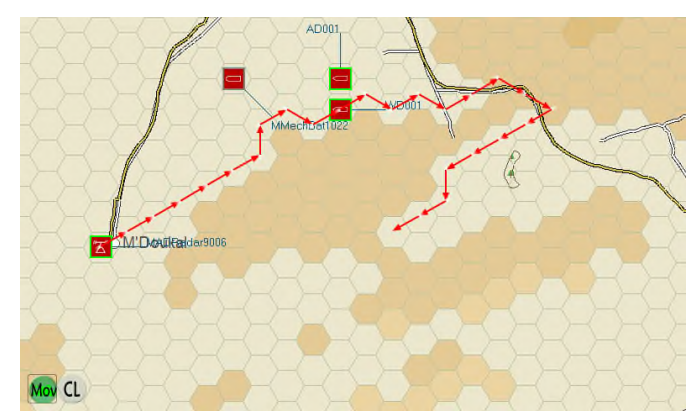

Fig. 4 Effectiveness of trajectory planning.

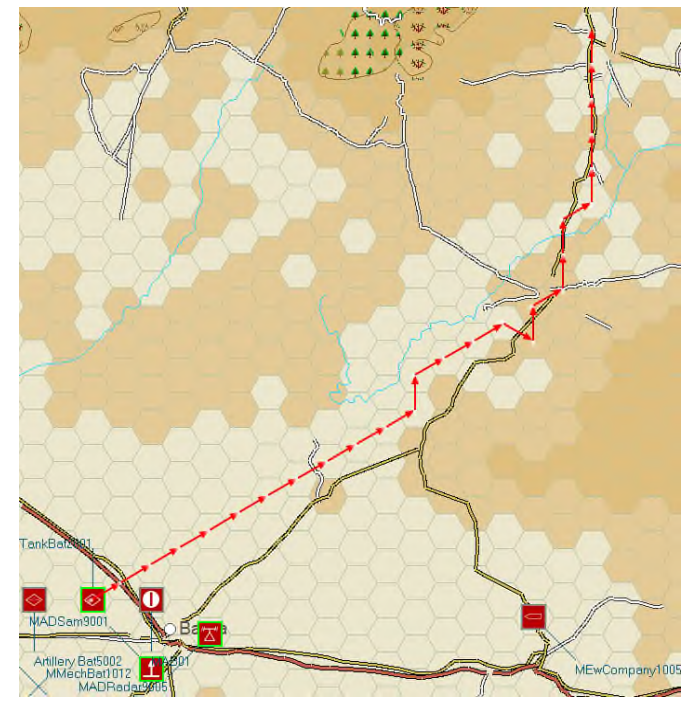

Fig. 5 Rationality of trajectory planning.

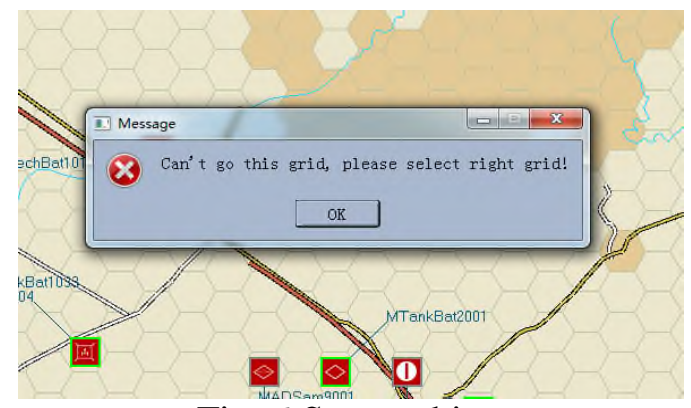

Fig. 6 System hint.

\section{Summary}

Since traditional Computer wargaming system only considers a few types of simple landform, maneuverable rules are simple, and simulation results are distrusted, this paper does trajectory planning based on real landform and real maneuverable rules, and takes a new heuristic function according to characteristic of hexagon grid. The simulation results show that the new algorithm is effective and reasonable. 


\section{References}

[1] N. Z. Yang, Fictitious troop training: wargaming and combat simulation, Beijing: Army Press, 2007.

[2] P. P. Peter, The Art of Wargaming, USA: Naval institute Press, 1990, pp. 8-9.

[3] J. X. Li, Q. Wu, Y. P. Li, Wargaming analysis, Changyin, 8 (2010) 22-23.

[4] X. F. Hu, J. Y. Yang, G. Y. Si, etal. War complex system simulation analysis and experimentation, Beijing: Press of National Defence University, 2008.

[5] W. Hu, The Research on Route Optimization of Military Power Maneuvering in Computer Wargame, Changsha: Graduate school of National University of Defense Technology, 2010.

[6] L. Condat, D. V. Van, T. Blu, Hexagonal versus Orthogonal lattices: A New Comparison Using Approximation Theory, Proceedings of the IEEE International Image Processing, Italy: IEEE, 9 (2005) 1116-1119.

[7] J. W. Liu, The Location and Maneuver Route Research of Logistics Center in Computer Wargame, School of National University of Defense Technology Changsha, 2010.

[8] Military Academy of Science edit and translate, Process of Determination of Tactics, Beijing, Military Science Press, 1997.

[9] Y. X. Ling, M. H. Ma, W. W. Yuan, etal. Combat Model and Simulation, National University of Defense Technology Press, 2006.

[10]D. Ross, Designing a system on system Wargame, U.S. Air Force Research Lab, 2006, pp. 6.

[11]P. Khosla, Real-Time Obstacle Avoidance Using Harmonic Potential Functions, Proc. IEEE Int. Conf. on Robotics and Automation, 1991. 